TITLE:

\title{
Polyhedral Structure of Submodular and Posi-modular Systems
}

$\operatorname{AUTHOR}(\mathrm{S})$ :

Nagamochi, Hiroshi; Ibaraki, Toshihide

\section{CITATION:}

Nagamochi, Hiroshi ... [et al]. Polyhedral Structure of Submodular and Posi-modular Systems. 数理解析研究所講究録 1998, 1068: 87-99

ISSUE DATE:

1998-10

URL:

http://hdl.handle.net/2433/62522

RIGHT: 


\title{
Polyhedral Structure of Submodular and Posi-modular Systems
}

\author{
Hiroshi Nagamochi and Toshihide Ibaraki \\ 永持 仁 茨木 俊秀 \\ Dept. of Applied Mathematics and Physics \\ Graduate School of Informatics \\ Kyoto University, Kyoto, Japan 606-8501 \\ \{naga,ibaraki\}@kuamp.kyoto-u.ac.jp
}

\begin{abstract}
Let $V$ be a finite set, and $\Re$ be the set of reals. We consider the polyhedron $P=\left\{z \in \Re_{-}^{V} \mid \sum_{i \in X} z(i) \leq f(X), \forall X \in 2^{V}\right\}$ for a system $(V, f)$ with an intersecting submodular and posi-modular set function $f: 2^{V} \mapsto \Re$, where $\Re_{-}^{V}$ denotes the set of $|V|$ dimensional nonpositive vectors. We first prove that there is a laminar family $\mathcal{X} \subseteq 2^{V}$ such that $P$ is characterized by $\left\{z \in \Re_{-}^{V} \mid \sum_{i \in X} z(i) \leq f(X), \forall X \in \mathcal{X}\right\}$. Based on this, we can solve in polynomial time the edge-connectivity augmentation problem with an additional constraint that the number of vertices to which new edges are incident is minimized.
\end{abstract}

\section{Introduction}

Let $V$ be a finite set, where $|V|$ is denoted by $n$. A singleton set $\{v\}$ may be written as $v$. For two subsets $X, Y \subseteq V$, we say that $X$ and $Y$ intersect each other if $X \cap Y \neq \emptyset, X-Y \neq \emptyset$ and $Y-X \neq \emptyset$. A family $\mathcal{X} \subset 2^{V}$ is called laminar if no two subsets in $\mathcal{X}$ intersect each other. Two intersecting sets $X$ and $Y$ are called crossing if $V-(X \cup Y) \neq \emptyset$ also holds.

Let $\Re$ (resp., $\Re_{+}$and $\Re_{-}$) be the set of reals (resp., nonnegative reals and nonpositive reals), and let $\Re^{V}$ (resp., $\Re_{+}^{V}$ and $\Re_{-}^{V}$ ) be the set of $n$-dimensional real vectors (resp., nonnegative real vectors and nonpositive real vectors) on a ground set $V$. A set function $f$ on $V$ is a function $f: 2^{V} \mapsto \Re$. For a vector $z \in \Re^{V}$ and a subset $X \subseteq V$, we denote $\sum_{i \in X} z(i)$ by $z(X)$. Such a function $z: 2^{V} \mapsto \Re$ is called modular. A function $f$ is called fully (resp., intersecting, crossing) submodular if it satisfies the following inequality

$$
f(X)+f(Y) \geq f(X \cap Y)+f(X \cup Y)
$$

holds for every (resp., intersecting, crossing) pair of sets $X, Y \subseteq V$. A function $f$ is called fully (resp., intersecting, crossing) supermodular if $-f$ is fully (resp., intersecting, crossing) submodular. An $f$ is called symmetric if $f(X)=f(V-X)$ holds for all $X \subseteq V$. In this paper, we call a function $f$ fully (resp., intersecting, crossing) posi-modular if

$$
f(X)+f(Y) \geq f(X-Y)+f(Y-X)
$$

holds for every (resp., intersecting, crossing) pair of sets $X, Y \subseteq V$ [8]. An $f$ is called fully (resp., intersecting, crossing) nega-modular if $-f$ is fully (resp., intersecting, crossing) posi-modular. Any modular function $z$ such that $z(i) \geq 0$ for all $i \in V$. is posi-modular. Also a symmetric fully submodular function $f$ is fully posi-modular. However, the converse is not generally true.

A pair $(V, f)$ of a finite set $V$ and a set function $f$ on $V$ is called a system. The optimization in a system $(V, f)$ has been much studied, such as: 


\section{Problem 1 (primal type): minimize $\Phi(z)$

$$
\begin{array}{lll}
\text { subject to } & z(X) \leq f(X) . & \text { for all } X \in 2^{V} \\
& 0 \leq z(i) \leq d(i) & \text { for all } i \in V
\end{array}
$$

(an additional constraint $z(V)=f(V)$ may also be imposed), where $\Phi(z): R^{V} \mapsto \Re$ is an objective function and $d \in \Re_{+}^{V}$ is a given constant vector. For fully submodular functions $f$, Problem 1 appears in many applications [5]. Given a system $(V, g)$, a dual type of this problem is stated as follows:

$$
\begin{aligned}
& \text { Problem } 2 \text { (dual type): minimize } \Phi(t) \\
& \text { subject to } \quad g(X) \leq t(X) \quad \text { for all } X \in 2^{V} \\
& 0 \leq t(i) \leq d(i) \quad \text { for all } i \in V
\end{aligned}
$$

(where we may also impose an additional constraint $t(V)=g(V)$ ). Problem 2 with a certain supermodular function $g$ appears in the edge-connectivity augmentation problem $[1,3]$ and the problem of computing the core of a convex game [10]. The above Problems 1 and 2 are generalized into the following common formulation. For two set functions $g$ and $f$ on $V$, and vectors $d_{1}, d_{2} \in \Re_{+}^{V}$, we consider:

$$
\begin{array}{lll}
\text { Problem } 3 \text { (mixed type): } & \text { minimize } & \Phi(z) \\
& \text { subject to } & g(X) \leq z(X) \leq f(X) \quad \text { for all } X \in 2^{V} \\
& & d_{1}(i) \leq z(i) \leq d_{2}(i) \quad \text { for all } i \in V .
\end{array}
$$

In this paper, we consider Problems 1-3 with intersecting submodular and posi-modular functions $f$ and $-g$.

Before going into details, let us explain an application of Problem 2 to the edge-connectivity augmentation problem. Let $N=(V, E, c)$ be an undirected complete network with a vertex set $V$, an edge set $E=V \times V$ and an edge weight function $c: E \mapsto \Re_{+}$. The cut function $f_{N}: 2^{V} \mapsto \Re_{+}$is defined by $f_{N}(X)=\sum\{c(e) \mid e=\{u, v\} \in E, u \in X, v \in V-X\}$ (where $f_{N}(\emptyset)=f_{N}(V)=0$ ). It is known (and easy to see) that the cut function $f_{N}$ is symmetric and fully submodular. The edge-connectivity augmentation problem asks to increase edge weights $c$ to obtain a $k$-edge-connected network $N^{\prime}$ (i.e., $f_{N^{\prime}}(X) \geq k$ holds for all $X \in 2^{V}-\{\emptyset, V\}$ ). Frank [3] introduced an additional constraint to this problem, the degree constraint: Given a vector $d \in \Re_{+}^{V}$, the output $k$-edge-connected network $N^{\prime}$ is required to satisfy $\sum_{e \in E(i)}\left(c^{\prime}(e)-c(e)\right) \leq d(i)$ for all $i \in V$, where $E(i)$ denotes the set of edges incident to a vertex $i$. The problem can be formulated as Problem 2 by using the following result.

Lemma $1[1,3]$ Given a network $N=(V, E, c)$, a constant $k \geq 0$, and a vector $t \in \Re_{+}^{V}$ such that

$$
f_{N}(X)+t(X) \geq k \text { for all } X \in 2^{V}-\{\emptyset, V\},
$$

there is a $k$-edge-connected network $N^{\prime}=\left(V, E, c^{\prime}\right)$ satisfying $\sum_{e \in E(i)}\left(c^{\prime}(e)-c(e)\right)=t(i)$ for all $i \in V$. Also, the $c^{\prime}$ can be chosen as integers if $c, t$ and $k \geq 2$ are integers and $t(V)=\sum_{i \in V} t(i)$ is an even integer. 
Notice that the total increase $\sum_{e \in E}\left(c^{\prime}(e)-c(e)\right)$ of weights is $\frac{1}{2} t(V)$. Therefore, in order to solve the edge-connectivity augmentation problem, we only need to find a vector $t \in \Re_{+}^{V}$ that minimizes $t(V)=\sum_{i \in V} t(i)$ among all vectors $t$ satisfying (3) (and $t(i) \leq d(i), i \in V$ if the degree constraint is imposed). Hence, by defining $\Phi(t)=\frac{1}{2} t(V)$ and a fully supermodular set function $g$ by $g(X)=k-f_{N}(X)$ for all $X \in 2^{V}$, we see that the smallest amount $\alpha(N, k)$ of new weights to be added to obtain a $k$-edge-connected network $N^{\prime}$ is given by the minimum value of $\Phi(t)$ over all $t \in \Re_{+}^{V}$ satisfying $g(X) \leq t(X)$ for all $X \in 2^{V}-\{\emptyset, V\}$ (and $t(i) \leq d(i), i \in V$ if the degree constraint is imposed). In the case of integer version, $\alpha(N, k)$ is given by $\left\lceil\frac{1}{2} \Phi(t)\right\rceil$. In any case, the problem of finding such a vector $t$ can be formulated as Problem 2 with these $\Phi, g$ and $d$.

In this paper, we first characterize the polyhedra of Problems 1-3 with intersecting submodular and posi-modular functions $f$ and $-g$, and then present a combinatorial algorithm for solving Problem 3 with a linear function $\Phi(t)$ (and assuming a further restriction on $g$ ). Note that Problem 3 is more general than Problem 2 in the sense that it allows additional constraints $z(X) \leq f(X), X \in 2^{V}$. This enables us to solve in polynomial time the edge-connectivity augmentation problem with a more general degree constraint that, for each subset $X \subset V$, the total increase of degrees in $X$ in the resulting network $N^{\prime}$ is bounded by a given constant $f(X)$.

We also show that Problem 2 can be solved for an objective function $\Phi(t)=\mid\{i \in V \mid t(i)>$ $0\} \mid$ in $O\left(n^{3}\right)$ function value oracle calls. Based on this, we can solve in polynomial time the problem of augmenting edge-connectivity of a network so as to minimize the number of vertices having edges whose weights are increased.

The paper is organized as follows. In Section 2, we characterize the polyhedron of Problem 2, and give algorithms for solving Problems 1-3. In Section 3, we characterize all the extreme points of the base polyhedron of Problem 2, and discuss a relation to the core of a convex game.

\section{Polyhedral Structures and Problems 1 - 3}

A polyhedron of a system $(V, f)$ is defined by

$$
P(f)=\left\{z \in \Re^{V} \mid z(X) \leq f(X), \forall X \in 2^{V}-\{\emptyset, V\}\right\},
$$

where $X=\emptyset$ and $V$ are not considered in the definition, and a base polyhedron of $(V, f)$ by

$$
B(f)=\{z \in P(f) \mid z(V)=f(V)\}
$$

where possibly $B(f)=\emptyset$. Let $P_{-}(f)$ and $B_{-}(f)$ denote $P(f) \cap \Re_{-}^{V}$ and $B(f) \cap \Re_{-}^{V}$, respectively, and let $P_{+}(f)$ and $B_{+}(f)$ denote $P(f) \cap \Re_{+}^{V}$ and $B(f) \cap \Re_{+}^{V}$, respectively. For two set functions $f_{1}$ and $f_{2}$ on $V$, we denote by $\left(f_{1}-f_{2}\right)$ the set function $f^{\prime}$ with $f^{\prime}(X)=f_{1}(X)-f_{2}(X)$ for all $X \in 2^{V}$.

We say that a subset $X \subset V$ separates $x$ and $y$ if $|\{x, y\} \cap X|=1$. For a submodular system $(V, f)$, an ordered pair $(x, y)$ of elements in $V$ is called a pendant pair if $f(x) \leq f(X)$ holds for all sets $X \subset V$ that separate $x$ and $y$. 
Lemma 2 [8] For an intersecting submodular and posi-modular set function $f$ on $V$ (where $n=|V| \geq 2)$, there exists a pendant pair $(x, y)$. Furthermore, such a pendant pair can be obtained by using $O\left(n^{2}\right)$ function value oracle calls.

\subsection{Polyhedral Structure of $P_{-}(f)$}

In this section, we first consider the set of all feasible vectors to Problem 2, where we assume that $g$ is an intersecting supermodular and nega-modular set function and a vector $d \in \Re_{+}^{V}$ is given by $d(i)=+\infty(i \in V)$. In this case, $f=-g$ is intersecting submodular and posi-modular. Then a vector $t$ is feasible to Problem 2 if and only if $-t \in P_{-}(f)$ holds for a system $(V, f)$.

We now prove that, given a system $(V, f)$ with an intersecting submodular and posi-modular set function $f$, there is a laminar family $\mathcal{X} \subseteq 2^{V}-\{\emptyset, V\}$ that characterizes $P_{-}(f)$ as follows.

$$
P_{-}(f)=P_{-}(f ; \mathcal{X})
$$

where we use notations $P(f ; \mathcal{X})=\left\{z \in \Re^{V} \mid z(X) \leq f(X)\right.$ for all $\left.X \in \mathcal{X}\right\}$ and $P_{-}(f ; \mathcal{X})=$ $P(f ; \mathcal{X}) \cap \Re_{-}^{V}$.

Given an intersecting submodular and posi-modular set function $f$ on $V$, we compute the above laminar family $\mathcal{X}$ as follows. Initially we set $\mathcal{X}:=\emptyset$ and $z(i):=0$ for all $i \in V$. Then, for each $i \in V$, we check whether $z(i) \leq f(i)$ (i.e., $f(i) \geq 0$ ) holds or not. If $f(i)<0$ then we reset $z(i)$ by $z(i):=f(i)$ and add $\{i\}$ to $\mathcal{X}$. Now $z(i) \leq f(i)$ (i.e., $(f-z)(i) \geq 0$ ) holds for all $i \in V$. Note that $f-z$ remains to be intersecting submodular and posi-modular. Hence there is a pendant pair $(x, y)$ in system $(V, f-z)$ by Lemma 2 , for which any cut $X$ separating $x$ and $y$ satisfies $z(X) \leq f(X)$. Then we can contract $x$ and $y$ into a single element $x^{*}$ without losing any cut $X$ that satisfies $z(X)>f(X)$. After this contraction, we check whether the new element $x^{*}$ satisfies $(f-z)\left(x^{*}\right) \geq 0$. If $(f-z)\left(x^{*}\right)<0$, then we add to $\mathcal{X}$ the set $X^{*}$ of all elements which have been contracted into $x^{*}$ so far, and decrease $z(i)$ of some $i \in X^{*}$ so that $z\left(X^{*}\right)=f\left(X^{*}\right)$ holds (where more than one $z(i)$ may be decreased as long as $z\left(X^{*}\right)=f\left(X^{*}\right)$ is satisfied). (If $(f-z)\left(x^{*}\right) \geq 0$, no $z(i)$ is changed.) Then we repeat finding a new pendant pair and contracting them into a single element in the resulting system, until the system has only one element. The entire algorithm is described as follows.

\section{Algorithm LAMINAR}

Input: A system $(V, f)$ with an intersecting submodular and posi-modular set

function $f$ on $V$, where $n=|V| \geq 2$.

Output: A vector $z \in P_{-}(f)$, and a laminar $\mathcal{X}$ of $V$ satisfying (3).

$1 \mathcal{X}:=\emptyset ; z(i):=0$ for all $i \in V$;

2 For each $i \in V$, if $f(i)<0$ then $z(i):=f(i)$ and $\mathcal{X}:=\mathcal{X} \cup\{\{i\}\}$;

3 for $i:=1$ to $n-1$ do

$4 \quad$ Find a pendant pair $(x, y)$ in $\left(V^{\prime}, f-z\right)$;

$5 \quad$ Let $\left(V^{\prime}, f-z\right)$ again denote the system obtained from the current $\left(V^{\prime}, f-z\right)$

by contracting $x$ and $y$ into a single element $x^{*}$;

6

if $(f-z)\left(x^{*}\right)<0$ then

$7 \quad$ Let $X^{*}$ be the set of all elements of $V$ which have been contracted to $x^{*}$; 
Clearly, LAMINAR runs in $O\left(n^{3}\right)$ function value oracle calls, as the pendant pair in line 4 can be found in $O\left(n^{2}\right)$ function value oracle calls by Lemma 2. Note that the vector $z$ output by LAMINAR may not be unique because there are many ways of decreasing $z(i), i \in X^{*}$ in line 8. Let OUTPUT $(f)$ denote the set of all vectors $z$ that can be output by LAMINAR for a given input $f$.

For a laminar family $\mathcal{X} \subseteq 2^{V}$ on $V$, a subset $Y \in \mathcal{X}$ is called a child of a subset $X \in \mathcal{X}$ (and the $X$ is called the parent of $Y$ ) if $Y \subset X$ and there is no other subset $Y^{\prime} \in \mathcal{X}$ with $Y \subset Y^{\prime} \subset X$. For a subset $X \in \mathcal{X}$, let $\operatorname{ch}(X)$ denote the set of children of $X$, and $p a(X)$ denote the parent of $X($ possibly $p a(X)=\emptyset)$.

Let $\mathcal{X}$ be a family of subsets of $V$ output by LAMINAR, which is clearly laminar. We represent $\mathcal{X}$ by a rooted tree as follows. Define the laminar family $\mathcal{V}=\mathcal{X} \cup\{V\} \cup\{\{i\} \mid i \in V\}$ and define the tree $T=(\mathcal{V}, \mathcal{E})$ on $\mathcal{V}$, where the parent-child relation in the tree is given by $p a(X)$ and $\operatorname{ch}(X)$. Clearly $V$ is the root of $T$. Define $f^{\prime}: \mathcal{V} \mapsto \Re$ by $f^{\prime}(X)=0$ if $X=\{i\}$ and $f(i) \geq 0$; $f^{\prime}(X)=f(X)$ otherwise. From the behavior of LAMINAR, the next properties are observed.

Lemma 3 For a system $(V, f)$ with an intersecting submodular and posi-modular set function $f$ on $V$ with $|V| \geq 2$, let $z$ and $\mathcal{X}$ be the vector and the laminar family output by algorithm LAMINAR. Let the tree $T$ be defined as above. Then:

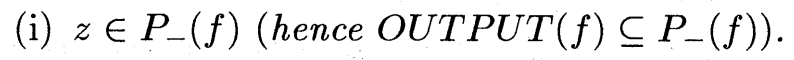

(ii) For each non-root vertex $X$ in $T, f^{\prime}(X)<\sum_{Y \in \operatorname{ch}(X)} f^{\prime}(Y)$ holds.

Now we prove (3). Clearly, $P_{-}(f ; \mathcal{X}) \supseteq P_{-}(f)$.

Theorem 1 For a system $(V, f)$ with an intersecting submodular and posi-modular set function $f$ on $V$ with $|V| \geq 2$, let $\mathcal{X}$ be the laminar family output by algorithm LAMINAR. Then:

(i) $P_{-}(f)=P_{-}(f ; \mathcal{X})$.

(ii) $B_{-}(f) \neq \emptyset$ if and only if $f^{\prime}(V) \leq \sum_{Y \in \operatorname{ch}(V)} f^{\prime}(Y)$ holds.

Proof: (i) We prove that any vector $z^{*} \in P_{-}(f ; \mathcal{X})$ also belongs to $P_{-}(f)$. By Lemma $3(\mathrm{i})$, it is sufficient to show that if $z^{*} \in P_{-}(f ; \mathcal{X})$ then there is a vector $z^{\prime} \in O U T P U T(f)$ such that $z^{*}(i) \leq z^{\prime}(i)$ for all $i \in V$. (This implies $z^{*} \in P_{-}(f)$.) Suppose that the execution of LAMINAR outputs $\mathcal{X}$. Assume that, in this execution LAMINAR also outputs $z_{0} \in \operatorname{OUTPUT}(f)$. We show that this execution can be modified so that it outputs $z^{\prime}$ for which $z^{*} \leq z^{\prime}$ holds. Now after executing line $2, z(i)=f(i) \geq z^{*}(i)$ holds if $f(i)<0$, and $z(i)=0 \geq z^{*}(i)$ holds if 
$f(i) \geq 0\left(\right.$ since $z^{*}(i) \leq \min \{f(i), 0\}$ for all $\left.i \in V\right)$. Thus, $z^{*} \leq z$ holds after line 2. We show by induction that a vector $z$ with $z^{*} \leq z$ can be chosen in line 8 during each iteration of the for-loop. Assume that $(f-z)\left(x^{*}\right)<0$ holds in line 6 at an iteration of the for-loop. Then in line 8 , the values $z(i)$ for $i \in X^{*}$ are decreased so that the resulting $z$ satisfies $(f-z)\left(X^{*}\right)=0$. By inductive hypothesis, we assume $z^{*} \leq z$. By $z^{*} \in P_{-}(f ; \mathcal{X})$, we have $z^{*}\left(X^{*}\right) \leq f\left(X^{*}\right)$. Since $z^{*}(i) \leq z(i)$ holds for all $i \in X^{*}$, we can decrease these $z(i), i \in X^{*}$, so that the resulting $z$ satisfies $(f-z)\left(X^{*}\right)=0$ while maintaining $z^{*}(i) \leq z(i)$ for all $i \in X^{*}$. Note that, in the contracted system $\left(V^{\prime}, f-z\right), z(j)=z_{0}(j)$ holds for all $j \in V^{\prime}$, and we can choose the same pendant pair in $\left(V^{\prime}, f-z\right)$ which has been used to compute $z_{0}$. By applying this argument repeatedly, we see that the algorithm can output a vector $z^{\prime}=z \in P_{-}(f)$ with $z^{*} \leq z^{\prime}$, while outputting the same laminar family $\mathcal{X}$.

(ii) From (i), we have $B_{-}(f)=\left\{z \in \Re_{-}^{V} \mid z(V)=f(V)\right\} \cap P_{-}(f ; \mathcal{X})$. Thus (ii) is immediate from the definition of $B_{-}(f)$ and $\operatorname{ch}(V)$.

\subsection{Problem 2}

Based on Theorem 1, we can solve Problem 2, if it has a linear objective function $\Phi(t)=$ $\sum_{i \in V} w(i) t(i)$ defined by a cost vector $w \in \Re^{V}$. The proof for this case will be given in Section 2.4 under a more general setting of Problem 3. We consider another special case of Problem 2 when the objective function is given by

$$
\Phi(t)=|\{i \in V \mid t(i)>0\}| \text { (i.e., the number of nonzero entries). }
$$

By Theorem 1, we prove the next property (the proof is omitted).

Theorem 2 For an intersecting supermodular and nega-modular set function $g$ on $V$, and $a$ vector $d \in \Re_{+}^{V}$, Problem 2 with an objective function $\Phi(t)=|\{i \in V \mid t(i)>0\}|$ can be solved by using $O\left(n^{3}\right)$ function value oracle calls. If Problem 2 is feasible, then there is a feasible vector $t$ which minimizes $\Phi(t)$ and $\Phi^{\prime}(t)=\sum_{i \in V} t(i)$ at the same time. Such a solution $t$ also can be found by using $O\left(n^{3}\right)$ function value oracle calls.

Proof: Let $\mathcal{X} \subseteq 2^{V}$ be the laminar family obtained from system $(V, f=-g)$ by applying Theorem 1. Note that if $-d(X)>f(X)$ for some $X \in 2^{V}$, then there is no vector $z$ satisfying $-d(X) \leq z(X) \leq f(X)$. We either construct a vector $z \in P_{-}(f ; \mathcal{X}) \cap\left\{z \in \Re_{-}^{V} \mid-d \leq z\right\}$ which minimizes the number of nonzero entries, or conclude $P_{-}(f ; \mathcal{X}) \cap\left\{z \in \Re_{-}^{V} \mid-d \leq z\right\}=\emptyset$ by detecting an $X$ with $-d(X)>f(X)$. For each $X \in \mathcal{X}$, call an $|X|$-dimensional vector $z_{X}$ on $X$ $X$-feasible if it satisfies $z_{X}(X) \leq f(X)$ and $-d(i) \leq z_{X}(i)$ for all $i \in X$, and let $\nu(X)$ denote the minimum number of nonzero entries in an $X$-feasible vector $z_{X}$ on $X$. An $X$-feasible vector $z_{X}$ on $X$ is called $X$-optimal if $\left|\left\{i \in X \mid z_{X}(i)<0\right\}\right|=\nu(X)$.

For a minimal subset $X \in \mathcal{M}(\mathcal{X})$, an $X$-optimal vector $z_{X}$ is easily obtained as follows. Let $X=\left\{i_{1}, i_{2}, \ldots, i_{|X|}\right\}$ satisfy $d\left(i_{1}\right) \geq d\left(i_{2}\right) \geq \cdots \geq d\left(i_{|X|}\right)$ without loss of generality. Then $\nu(X)$ is given by the smallest $p$ such that $-\sum_{1 \leq j \leq p} d\left(i_{j}\right) \leq f(X)$, and an $X$-optimal vector $z_{X}$ is given by $z_{X}\left(i_{j}\right)=-d\left(i_{j}\right)$ for $1 \leq j<p, z_{X}\left(i_{p}\right)=\sum_{1 \leq j \leq p-1} d\left(i_{j}\right)+f(X)$, and $z_{X}\left(i_{j}\right)=0$ for $p+1 \leq j \leq|X|$. 
Now assume that, for each minimal subset $X \in \mathcal{M}(\mathcal{X})$, an $X$-optimal vector $z_{X}$ is computed in the above manner. Let $z \in \Re_{-}^{V}$ be the vector such that $z(i)=z_{X}(i)$ if $i$ belongs to some $X \in \mathcal{M}(\mathcal{X}) ; z(i)=0$ otherwise. Let $\mathcal{X}^{\prime}:=\mathcal{X}-\mathcal{M}(\mathcal{X})$, and consider a subset $Y \in \mathcal{M}\left(\mathcal{X}^{\prime}\right)$.

Notice that for each $X \in \operatorname{ch}(Y)$, there are at least $\nu(X)$ nonzero entries among $i \in X$ to satisfy $X$-feasibility. Thus, $\nu(Y) \geq \sum_{X \in c h(Y)} \nu(X)$. Then, a $Y$-optimal vector $z_{Y}$ is obtained from the current $z$ by increasing nonzero entries in $Y$ and then the fewest number of zero entries in $Y$. More precisely, let $Y^{\prime}=\left\{i_{1}, \ldots, i_{q}\right\}$ be the set of $i \in Y$ with $z(i)<.0$, and we perform $z\left(i_{j}\right):=z\left(i_{j}\right)-\min \left\{z\left(i_{j}\right)+d\left(i_{j}\right), z(Y)-f(Y)\right\}$ in the order of $j=1, \ldots, q$. Then let $Y-Y^{\prime}=\left\{i_{q+1}, \ldots, i_{|Y|}\right\}$ be the set of zero entries in $Y$, where we assume $d\left(i_{q+1}\right) \geq \cdots \geq d\left(i_{|Y|}\right)$. Choose the smallest $p$ such that $-\sum_{q+1 \leq j \leq p} d\left(i_{j}\right)+z\left(Y^{\prime}\right) \leq f(Y)$, and set $z_{X}\left(i_{j}\right):=-d\left(i_{j}\right)$ for $q+1 \leq j<p, z_{X}\left(i_{q}\right):=\sum_{q+1 \leq j \leq p-1} d\left(i_{j}\right)+f(Y)-z\left(Y^{\prime}\right)$, and $z_{X}\left(i_{j}\right):=0$ for $q+1 \leq j \leq|Y|$. (If such $p$ cannot be chosen, then $-d(Y)>f(Y)$ holds for this $Y$, indicating the infeasibility of the problem.)

Therefore, by applying this procedure recursively from minimal subsets to maximal subsets in $\mathcal{X}$ as long as such $p$ can be chosen (otherwise the problem is infeasible), we can obtain a vector $z \in P_{-}(f)$ such that $z_{X}$ (defined by $\left.z_{X}(i)=z(i), i \in X\right)$ is $X$-optimal for every maximal subset $X \in \mathcal{X}$. The algorithm is described as follows.

\section{Procedure NONZERO}

$1 \quad \mathcal{X}^{\prime}:=\mathcal{X} ; z(i):=0$ for all $i \in V$;

2 while $\mathcal{X}^{\prime} \neq \emptyset$ do

3 Choose a minimal subset $X$ in the current $\mathcal{X}^{\prime}$;

4 while $z(X)>f(X)$ and there is an element $i \in X$ with $-d(i)<z(i)<0$ do

$5 \quad$ Update $z(i)$ by $z(i)-\min \{z(i)+d(i), z(X)-f(X)\}$

6 end $/ *$ while $* /$

$7 \quad$ while $z(X)>f(X)$ do

$8 \quad$ Choose $i \in X$ such that $z(i)=0$ and $d(i)$ is maximum;

$9 \quad$ if there is no such $i$ then halt by concluding that there is no feasible vector end $\{$ if $\}$

$10 \quad$ Set $z(i):=\max \{f(X)-z(X),-d(i)\}$

11 end $/ *$ while $* /$

/* now $z(X)=f(X)$ holds */

$12 \mathcal{X}^{\prime}:=\mathcal{X}^{\prime}-X$

13 end /* while */

By applying this and Lemma 1 to the degree constrained edge-connectivity augmentation problem of a graph to minimize the number of vertices whose incident edges have increased weights, we obtain the next.

Theorem 3 For a complete network $N=(V, E=V \times V, c), k \geq 2$ and $d \in \Re_{+}^{V}$, where all $c(e), k$ and $d(i)$ are integers, let $c^{\prime}(\geq c)$ be a new integer-valued edge weight function such that $N^{\prime}=\left(V, E, c^{\prime}\right)$ is $k$-edge-connected under the degree constraint that new degree of each vertex $i \in V$ is at most $d(i)$. There is a $c^{\prime}$ that simultaneously minimizes (i): the number of 
vertices to which an edge with increased weight is incident and (ii) the total amount of increase $\sum_{e \in E}\left(c^{\prime}(e)-c(e)\right)$. Such a $c^{\prime}$ can be found in $O\left(\left(n m+n^{2} \log n\right) \log n\right)$ time, where $n=|V|$ and $m$ is the number of edges of positive weights in $N$.

Proof: (Sketch) Let $f_{N}$ be the cut function of $N$ defined by $f_{N}(X)=\sum\{c(e) \mid e=(i, j) \epsilon$ $E, i \in X, j \in V-X\}$. To find a $t \in \Re_{+}^{n}$ such that

$$
f_{N}(X)+t(X) \geq k \text { for all } X \in 2^{V}-\{\emptyset, V\},
$$

we compute a laminar family $\mathcal{X} \subset 2^{V}$ of Theorem 1 (i) for $f=f_{N}-k$ by using LAMINAR. For the cut function $f_{N}$, it is known that LAMINAR can be implemented to run in $O(n(m+n \log n))$ time [6]. Given such a laminar family $\mathcal{X}$, we can find $z=-t$ minimizing $|z(V)|$ and $|\{i \in V \mid z(i) \neq 0\}|$ at the same time under the condition (4), by procedure NONZERO in $O\left(n^{2} \log n\right)$ time. If $|t(V)|=|z(V)|$ is odd, we increase an arbitrary $t(i)$ by one. It is known [7] that, given a $t$ such that $|t(V)|$ is even and satisfies (4), there is a $k$-edge-connected network $N^{\prime}=\left(V, E, c^{\prime}\right)$ satisfying $c^{\prime} \geq c$ and $f_{N^{\prime}}(i)=f_{N}(i)+t(i)$ for all $i \in V$. Such $N^{\prime}$ can be obtained in $O\left(\left(n m+n^{2} \log n\right) \log n\right)$ time [7].

\subsection{Problem 1}

In this subsection, we consider polyhedra $P(f)$ and $P_{+}(f)$ for an intersecting submodular and posi-modular function $f$ on $V$, which appear in Problem 1 . However, we do not consider the constraint $z \leq d$, as this more general case will be considered in the next subsection as Problem 3 .

To generalize Theorem 1 to this case, we further assume that the set function $\hat{f}$ defined by

$$
\hat{f}(X)=f(X)-m_{f}(X) \text { for } X \in 2^{V}
$$

is intersecting submodular and posi-modular, where $m_{f}$ denotes the modular function on $V$ defined from $f$ by $m_{f}(i)=f(i)$ for all $i \in V$.

Remark: There is a non-modular function $f$ such that $f-m_{f}$ is also intersecting submodular and posi-modular. Consider a network $N=(V, E, c)$, and assume that its cut function $f_{N}$ satisfies $f_{N}(i)=k$ for all vertices $i \in V$ (i.e., $N$ is a $k$-regular edge-weighted graph). The cut function $f_{N}$ is intersecting submodular and posi-modular, and so is function $\hat{f}$ with $\hat{f}(X)=$ $f_{N}(X)-k, X \in 2^{V}$, where $\hat{f}(i)=0$ for all $i \in V$. Hence for any vector $a \in \Re_{+}^{V}$, the set function $f$ with $f(X)=\hat{f}(X)+a(X)\left(X \in 2^{V}\right)$ satisfies the property that $f-m_{f}$ is intersecting submodular and posi-modular.

Now we discuss how to compute a vector $z \in P_{+}(f)$. Let us consider $y=z-m_{f}$. Then $0 \leq z(X) \leq f(X)$ holds if and only if $0 \leq y(X)+m_{f}(X) \leq f(X)=\hat{f}(X)-m_{f}(X)$. Thus the problem is equivalent to finding a vector $y \in P(\hat{f}) \cap\left\{y \in \Re^{V} \mid y+m_{f} \geq 0\right\}$.

We first consider $P(f)$. Note that $P(\hat{f})=P_{-}(\hat{f})$ since $\hat{f}(i)=0$ holds for all $i \in V$. Therefore, $P(f)=\left\{z=y+m_{f} \mid y \in P_{-}(\hat{f})\right\}$. By applying Theorem 1 to system $(V, \hat{f})$, we obtain a laminar family $\mathcal{X}$ such that $P_{-}(\hat{f})=P_{-}(\hat{f} ; \mathcal{X})$, by using $O\left(n^{3}\right)$ function value oracle calls. Clearly, for any $z \in P(f ; \mathcal{X})$, we have $y=z-m_{f} \in P_{-}(\hat{f} ; \mathcal{X})=P_{-}(\hat{f})$, and hence $z \in P(f)$ (the converse is also clear). Thus, $P(f)=P(f ; \mathcal{X})$ holds. 
Next consider a vector $z \in P_{+}(f)$. From the above argument, it holds $P_{+}(f)=P(f) \cap \Re_{+}^{V}=$ $\left\{z=y+m_{f} \mid y \in P_{-}(\hat{f}), y \geq-m_{f}\right\}$. A vector $y \in P_{-}(\hat{f})$ satisfying constraint $y \geq-m_{f}$ (if any) can be easily computed. From these, we establish the next result.

Theorem 4 For a system $(V, f)$ with a set function $f$ on $V$ with $n=|V| \geq 2$ such that $f-m_{f}$ is intersecting submodular and posi-modular, there is a laminar family $\mathcal{X}$ such that $P(f)=P(f ; \mathcal{X})$. Such a family $\mathcal{X}$ and a vector $z \in P_{+}(f)$ (if any) can be found by $O\left(n^{3}\right)$ function value oracle calls.

\subsection{Problem 3}

In this subsection, we solve Problem 3 with a linear objective function $\Phi(z)=\sum_{i \in V} w(i) z(i)$ for a given vector $w \in \Re^{V}$.

Theorem 5 Let $g$ and $f$ be set functions on $V$, and $d_{1}, d_{2} \in \Re_{+}^{V}$ and $w \in \Re^{V}$. If $-g$ and $f-m_{f}$ are both intersecting submodular and posi-modular, then an optimal solution $z$ to Problem 3 with objective function $\Phi(z)=\sum_{i \in V} w(i) z(i)$ can be found by using $O\left(n^{3}\right)$ function value oracle calls and by solving a minimum cost flow problem with $O(n)$ vertices and arcs.

Proof: By Theorem 1, there is a laminar family $\mathcal{X}_{1}$ such that

$$
\left\{z \in \Re_{+}^{V} \mid g(X) \leq z(X) \text { for all } X \in \mathcal{X}_{1}\right\}=\left\{z \in \Re_{+}^{V} \mid g(X) \leq z(X) \text { for all } X \in 2^{V}-\{\emptyset, V\}\right\} .
$$

Analogously, by Theorem 4 , there is a laminar family $\mathcal{X}_{2}$ such that

$$
\left\{z \in \Re^{V} \mid z(X) \leq f(X) \text { for all } X \in \mathcal{X}_{2}\right\}=\left\{z \in \Re^{V} \mid z(X) \leq f(X) \text { for all } X \in 2^{V}-\{\emptyset, V\}\right\} .
$$

Thus, the problem is restated as

$$
\begin{array}{lll}
\text { minimize } & \Phi(z)=\sum_{i \in V} w(i) z(i) & \\
\text { subject to } & g(X) \leq z(X) & \text { for all } X \in \mathcal{X}_{1}^{\prime} \\
& z(X) \leq f(X) & \text { for all } X \in \mathcal{X}_{2}^{\prime} \\
& d_{1}(i) \leq z(i) \leq d_{2}(i) & \text { for all } i \in V,
\end{array}
$$

where $\mathcal{X}_{i}^{\prime}=\mathcal{X}_{i} \cup\{\emptyset, V\}$ for $i=1,2$. Denote this problem by $P\left(d_{1}, d_{2}, w, \mathcal{X}_{1}^{\prime}, \mathcal{X}_{2}^{\prime}, g, f\right)$.

The problem can be formulated as the minimum cost flow problem in the following directed network $\mathcal{N}=\left(\mathcal{G}=(\mathcal{V}, \mathcal{A}), \underline{c}, \bar{c}, w^{\prime}\right)$, where $\mathcal{G}$ is a digraph with a vertex set $\mathcal{V}$, an arc set $\mathcal{A}$, lower and upper capacity functions $\underline{c}, \bar{c}: \mathcal{A} \mapsto \Re^{+}$and a cost function $w^{\prime}: \mathcal{A} \mapsto \Re$. The vertex set $\mathcal{V}$ in the digraph $\mathcal{G}$ consists of $\mathcal{V}_{1}$ and $\mathcal{V}_{2}$. The $\mathcal{V}_{1}$ contains a sink $t$ and vertices $v_{X}$, associated with subsets $X \in \mathcal{X}_{1}^{\prime}$. Similarly, $\mathcal{V}_{2}$ contains a source $s$ and vertices $u_{X}$, associated with subsets $X \in \mathcal{X}_{2}^{\prime}$. The arc set $\mathcal{A}$ consists of the following arc sets $\mathcal{A}_{1}, \mathcal{A}_{2}$ and $\mathcal{A}_{3}$. For two vertices $v, v^{\prime} \in \mathcal{V}_{1}, \mathcal{A}_{1}$ contains an arc $\left(v, v^{\prime}\right)$ if and only if $v=v_{X}$ and $v^{\prime}=v_{X^{\prime}}$ hold for $X, X^{\prime} \in \mathcal{X}_{1}^{\prime}$ such that $p a(X)=X^{\prime}$ (or $X=V$ and $v^{\prime}=t$ ). Similarly, for two vertices $u, u^{\prime} \in \mathcal{V}_{2}, \mathcal{A}_{2}$ contains an $\operatorname{arc}\left(u, u^{\prime}\right)$ if and only if $u=u_{X}, u^{\prime}=u_{X^{\prime}}$ for $X, X^{\prime} \in \mathcal{X}_{1}^{\prime}$ such that $p a\left(X^{\prime}\right)=X$ (or $u=s$ and $\left.X^{\prime}=V\right)$. Finally, $\mathcal{A}_{3}$ consists of arcs $e_{i}=(u, v)$, associated with the elements $i \in V$ such that $v=v_{X} \in \mathcal{V}_{1}$ for a minimal $X$ with $i \in X \in \mathcal{X}_{1}^{\prime}$ and $u=u_{Y} \in \mathcal{V}_{2}$ for a minimal $Y$ with $i \in Y \in \mathcal{X}_{2}^{\prime}$.

The lower and upper capacity functions $\underline{c}, \bar{c}: \mathcal{A} \mapsto \Re_{+}^{V}$ and cost function $w^{\prime}: \mathcal{A} \mapsto \Re^{V}$ are defined by 


$$
\begin{aligned}
& \underline{c}(e)=g(X), \bar{c}(e)=+\infty, w^{\prime}(e)=0 \text { for } e=\left(v, v^{\prime}\right) \in \mathcal{A}_{1} \text { with } v=v_{X} \text { and } X \in \mathcal{X}_{1}^{\prime}, \\
& \underline{c}(e)=0, \bar{c}(e)=f(X), w^{\prime}(e)=0 \text { for } e=\left(u, u^{\prime}\right) \in \mathcal{A}_{2} \text { with } u^{\prime}=u_{X} \text { and } X \in \mathcal{X}_{2}^{\prime}, \\
& \underline{c}\left(e_{i}\right)=d_{1}(i), \bar{c}\left(e_{i}\right)=d_{2}(i), w^{\prime}\left(e_{i}\right)=w(i) \text { for } e_{i} \in \mathcal{A}_{3} .
\end{aligned}
$$

It is not difficult to see that any feasible flow $z \in \Re_{+}^{\mathcal{A}}$ in $\mathcal{N}$ gives a vector $z^{\prime}$ with $z^{\prime}(i)=z\left(e_{i}\right)$, $i \in V$ which is feasible to $P\left(d_{1}, d_{2}, w, \mathcal{X}_{1}^{\prime}, \mathcal{X}_{2}^{\prime}, g, f\right)$. Also, if $z$ is a minimum cost flow $z \in \Re_{+}^{\mathcal{A}}$ in $\mathcal{N}$, then the corresponding vector $z^{\prime}$ is optimal to the problem. This proves the theorem.

\section{Extreme points of base polyhedron}

In this section, we characterize all extreme points of a base polyhedron $B_{-}(f)$ defined for an intersecting submodular and posi-modular set function $f$. We then show some relation of the result to a core in a convex game.

\subsection{All extreme points of $B_{-}(f)$}

Let $\Pi_{n}$ be the set of all permutations of $(1,2, \ldots, n)$. For a subset $P \subseteq \Re_{-}^{V}$ and a permutation $\pi \in \Pi_{|V|}$, a vector $z \in \Re_{-}^{V}$ is called lexicographically $\pi$-minimal ( $\pi$-minimal, for short) in $P$ if there is no other vector $z^{\prime} \in P$ which is lexicographically smaller than $z$ with respect to $\pi$; i.e., there is no $j$ such that $z^{\prime}(\pi(i))=z(\pi(i))$ for $i=1,2, \ldots, j-1$ and $z^{\prime}(\pi(j))<z(\pi(j))$. Let $L(f)$ be the set of $\pi$-minimal vectors in $B_{-}(f)$ for all $\pi \in \Pi_{n}$, and $E P(f)$ be the set of all extreme points in $B_{-}(f)$. Based on Theorem 1, we can show the next result.

Theorem 6 Let $(V, f)$ be a system with an intersecting submodular and posi-modular set function $f$ on $V$ with $n=|V| \geq 2$. If $B_{-}(f) \neq \emptyset$, then $L(f)=E P(f)$ holds.

Proof: It is clear that any $\pi$-minimal vector in $B_{-}(f)$ is an extreme point of $B_{-}(f)$, i.e., $L(f) \subseteq E P(f)$. We show $L(f) \supseteq E P(f)$. For this, it suffices to show that for any cost $w \in \Re^{V}$ (with $w(i) \neq w(j)$ for $i \neq j$ ), a vector $z \in B_{-}(f)$ that minimizes $w z$ belongs to $L(f)$. Let $\mathcal{X}$ be a laminar family output by algorithm LAMINAR for this system $(V, f)$. By Theorem 1 , $B_{-}(f)=\left\{z \in \Re_{-}^{V} \mid z(V)=f(V)\right\} \cap P_{-}(f ; \mathcal{X})$. Without loss of generality, we assume that $w(1)>w(2)>\cdots>w(n)$. Let $z^{*}$ be a $\pi$-minimal vector in $L(f)$ for $\pi=(1,2, \ldots, n)$. We derive a contradiction by assuming that there is a vector $z^{\prime} \in B(f)-\left\{z^{*}\right\}$ that minimizes $w z$ and satisfies $w z^{\prime} \leq w z^{*}$. Since $z^{\prime} \neq z^{*}$ and $z^{*}$ is $\pi$-minimal, there is a $j$ such that $z^{*}(i)=z^{\prime}(i)$ for all $i=1,2, \ldots, j-1$ and $z^{*}(j)<z^{\prime}(j)$. We choose the subset $X$ with the smallest $|X|$ among these $X \in \mathcal{X} \cup\{V\}$ satisfying $j \in X$ and $z^{*}(X)=f(X)$ (such $X$ exists by $z^{*}(V)=f(V)$ ). Since $z^{*}(X)=f(X) \geq z^{\prime}(X)$ holds, $X$ contains an element $h>j$ with $z^{*}(h)>z^{\prime}(h)$. We claim that such an $h$ can be chosen so that no $Y \in \mathcal{X}$ with $h \in Y \subset X$ satisfies $z^{\prime}(Y)=f(Y)$. To prove this, assume otherwise;i.e., for each $h \in X$ with $z^{*}(h)>z^{\prime}(h), \mathcal{X}$ contains a cut $Y_{h}$ with $h \in Y_{h} \subset X$ and $z^{\prime}\left(Y_{h}\right)=f\left(Y_{h}\right)$. Let $\mathcal{Y}$ be the set of all maximal such cuts $Y_{h}$ (hence two $Y, Y^{\prime} \in \mathcal{Y}$ are pairwise disjoint). Note that $j \notin \cup_{Y \in \mathcal{Y}} Y$ holds by the choice of $X$. Then $z^{\prime}(i) \geq z^{*}(i)$ for all $i \in X-\cup_{Y \in \mathcal{Y}} Y$, and in particular $z^{\prime}(i)>z^{*}(i)$ if $i=j$. Hence, we have 
$z^{\prime}(X)=\sum_{Y \in \mathcal{Y}} f(Y)+z^{\prime}\left(X-\cup_{Y \in \mathcal{Y} Y} Y\right)>z^{*}(X)=f(X)$, a contradiction. Therefore, there exists a desired $h \in X$. In this case, we can choose an $\varepsilon>0$ such that the vector $z^{\prime \prime}$ defined by $z^{\prime \prime}(j)=z^{\prime}(j)-\varepsilon, z^{\prime \prime}(h)=z^{\prime}(h)+\varepsilon$ and $z^{\prime \prime}(i)=z^{\prime}(i)$ for all $i \in V-\{j, h\}$ belongs to $B(f)$. Clearly, $w z^{\prime}>w z^{\prime \prime}$ must hold by $h>j$ and assumption $w(h)<w(j)$. This, however, contradicts the minimumity of $w z^{\prime}$.

If $B_{-}(f) \neq \emptyset$ and $L(f)=E P(f)$, then we define the mean vector $\psi_{f}$ of all $\pi$-minimal vectors $z_{\pi}, \pi \in \Pi_{n}$ by $\psi_{f}=\frac{1}{n !} \sum\left\{z_{\pi} \mid \pi \in \Pi_{n}\right\}$, where possibly $z_{\pi}=z_{\pi^{\prime}}$ holds for two permutations $\pi, \pi^{\prime} \in \Pi_{n}$. Again by Theorem 1, we can show that, for an intersecting submodular and posimodular set function $f$, the mean vector $\psi_{f}$ can be efficiently computed from the laminar family $\mathcal{X}$.

Theorem 7 For a system $(V, f)$ with an intersecting submodular and posi-modular set function $f$ on $V$ with $n=|V| \geq 2$, let $\mathcal{X}$ be the laminar family output by algorithm LAMINAR. Then the mean vector $\psi_{f}$ of all lexicographically minimal vectors in $B_{-}(f)$ can be computed from $\mathcal{X}$ in $O\left(n^{2}\right)$ time.

\subsection{A relation to a convex game}

A cooperative game in the game theory is defined by a pair $(V, g)$ of a set $V$ of players and a nonnegative set function $g$ on $V$, where $g$ is called the characteristic function of the game and satisfies $g(\emptyset)=0$. Several solution concepts such as core, Shapley value, $\tau$-value and others have been proposed. The core of a game $(V, g)$ is the set $C O R E(g)$ of nonnegative vectors $z \in \Re_{+}^{V}$ such that $z(X) \geq g(X)$ for all $X \in 2^{V}$ and $z(V)=g(V)$. In other words, it can be defined by $\operatorname{CORE}(g)=\left\{-z^{\prime} \mid z^{\prime} \in B_{-}(-g)\right\}$ for a system $(V,-g)$. Note that $\operatorname{CORE}(g)$ is always a convex set. The problem of testing whether a convex game $(V, g)$ has a nonempty $\operatorname{CORE}(g)$ can be solved by computing the minimum value $\Phi(z)=\sum_{i \in V} z(i)$ in Problem 2 with $d=+\infty$.

The Shapley value $\phi_{g} \in \Re^{V}$ is a solution concept proposed by Shapley [9], which is defined by

$$
\phi_{g}(i)=\sum_{S \subseteq V: i \in S} \frac{(|S|-1) !(n-|S|) !}{n !}[g(S)-g(S-i) \text { for each } i \in V .
$$

A game $(V, g)$ is called convex if $g$ is a fully supermodular set function on $V$. Several structural properties have been studied for a convex game. In this section, we consider a game $(V, h)$ with an intersecting supermodular and nega-modular function $h$ on $V$, which is slightly different from a convex game $(V, g)$. We show that a game $(V, h)$ has a considerably different structure from that of a convex game. Let us review some structural properties of a convex game.

Theorem $8[2,10]$ For a convex game $(V, g), C O R E(g)$ is always nonempty. For any permutation $\pi \in \Pi_{n}$, the $\pi$-minimal vector $z_{\pi} \in \Re_{+}^{V}$ belongs to CORE $(g)$ and is given by $z_{\pi}(\pi(i))=$ $g(\{\pi(1), \pi(2), \ldots, \pi(i)\})-g(\{\pi(1), \pi(2), \ldots, \pi(i-1)\})$ for $i=1,2, \ldots, n$. Moreover, the set of all extreme points of CORE $(g)$ is given by the set of $\pi$-minimal vectors $z_{\pi}, \pi \in \Pi_{n}$.

We denote by $\psi_{g}$ the mean vector of all $\pi$-minimal vectors $z_{\pi}, \pi \in \Pi_{n}$, for a convex game $(V, g)$. 
Theorem 9 [9] For a convex game $(V, g)$, the Shapley value $\phi_{g} \in \Re^{V}$ is given by $\phi_{g}=\psi_{g}$.

As to computing the Shapley value, we easily observe the following intractability.

Lemma 4 For a convex game $(V, g)$, there is no algorithm that computes the Shapley value $\phi_{g} \in \Re^{V}$ by using less than $2^{n}-1$ function value oracle calls, where $n=|V|$.

Proof: Let us consider a convex game $(V, g)$ such that $g(X)=|X|^{2}+\varepsilon_{X}$ for $X \in 2^{V}$, where $\varepsilon_{X}=0$ for $X=\emptyset$ (by $g(\emptyset)=0$ ) and $0<\varepsilon_{X}<1$ is chosen for each $X$ independently. It is easy to check that the resulting $g$ is a fully supermodular set function on $V$. Note that the vector $\phi_{g} \in \Re^{V}$ changes its value if we change each $\varepsilon_{X}$, while fixing all other $\varepsilon_{Y}, Y \in 2^{V}-X$. Therefore, if a vector $\phi_{g} \in \Re^{V}$ is computed by using less than $2^{n}-1$ function value oracle calls, then there is a subset $X \in 2^{V}$ whose function value $g(X)$ has not been refereed. This is a contradiction because the above argument says that we cannot determine $\phi_{g}$ without knowing the value of $\varepsilon_{X}$.

Now let us consider the counter part of the above results in a game $(V, h)$ with an intersecting supermodular and nega-modular function $h$. By applying Theorems 1 and 6 to system $(V,-h)$, we have the next result.

Theorem 10 For a game $(V, h)$ with an intersecting supermodular and nega-modular function $h: 2^{V} \mapsto \Re_{+}, C O R E(h)$ is nonempty if and only if $h(V) \geq \sum_{Y \in \operatorname{ch}(V)} h(Y)$ holds, where ch $(V)$ $i s$ the set of maximal subsets $X$ in the laminar family $\mathcal{X}$ obtained from $(V,-h)$ by algorithm LAMINAR. Moreover, the set of all extreme points of CORE $(h)$ is given by the set of $\pi$-minimal vectors $z_{\pi}, \pi \in \Pi_{n}$.

From Theorem 7, we obtain the following.

Theorem 11 For a game $(V, h)$ with an intersecting supermodular and nega-modular function $h: 2^{V} \mapsto \Re_{+}$, assume that CORE $(h) \neq \emptyset$. Then the mean vector $\psi_{h}$ of all $\pi$-minimal vectors $z_{\pi}, \pi \in \Pi_{n}$, can be computed by using $O\left(n^{3}\right)$ function value oracle calls, where $n=|V|$.

Moreover, the mean vector $\psi_{g}$ is no longer equal to the Shapley value $\phi_{h}$, as shown by the next lemma.

Lemma 5 For a game $(V, h)$ with an intersecting supermodular and nega-modular function $h: 2^{V} \mapsto \Re_{+}$, there is no algorithm that computes the Shapley value $\phi_{h} \in \Re^{V}$ by using less than $2^{n}-1$ function value oracle calls, where $n=|V|$.

\section{Conclusion}

In this paper, we showed that, for an intersecting submodular and posi-modular set function $f$ on $V$, its polyhedron $P_{-}(f)$ is described by a set of inequalities $z(X) \leq f(X)$ such that $X$ is in a laminar family $\mathcal{X} \subseteq 2^{V}$. Furthermore, such a laminar family can be obtained combinatorially by $O\left(|V|^{3}\right)$ function value oracle calls. This significantly reduces the complexity of finding a 
vector $z$ in the polyhedron $P_{-}(f)$. As a result, we show that several optimization problems over the polyhedron have efficient combinatorial algorithms, and that the core and its mean vector of some cooperative game can be efficiently computed. It is left for the future research to widen the class of set functions to which similar algorithms are applicable.

\section{Acknowledgments}

This research was partially supported by the Scientific Grant-in-Aid from Ministry of Education, Science, Sports and Culture of Japan, and the subsidy from the Inamori Foundation.

\section{References}

[1] G.-R. Cai and Y.-G. Sun, The minimum augmentation of any graph to k-edge-connected graph, Networks, 19, 1989, 151-172.

[2] J. Edmonds, Submodular functions, matroids, and certain polyhedra, Proc. Calgary Int. Conference on Combinatorial Structures and Their Applications (R. Guy, H. Hanani, N. Sauer and J. Schönheim, eds., Gordon and Breach, New York), 1970, 69-87.

[3] A. Frank, Augmenting graphs to meet edge-connectivity requirements, SIAM J. Discrete Mathematics, 5, 1992, 25-53.

[4] A. Frank, Applications of submodular functions, in Surveys in Combinatorics, Keith Walker, ed., London Math. Soc. Lecture Notes Ser. 187, 1993, 85-136.

[5] T. Ibaraki and N. Katoh, Resource Allocation Problems - Algorithmic Approaches, Foundations of Computing Series, MIT Press, 1988.

[6] H. Nagamochi and T. Ibaraki, Computing edge-connectivity of multigraphs and capacitated graphs, SIAM J. Discrete Mathematics, 5, 1992, 54-66.

[7] H. Nagamochi and T. Ibaraki, Deterministic Õ $(\mathrm{nm})$ time edge-splitting in undirected graphs, Proceedings 28th ACM Symposium on Theory of Computing, 1996, 64-73 J. Combinatorial Optimization, 1, 1997, 5-46.

[8] H. Nagamochi and T. Ibaraki, A note on minimizing submodular functions, Information Processing Letters (to appear).

[9] L. S. Shapley, A value for n-person games, H. W. Kuhn and A. W. Tucker (eds), Contributions to the Theory of Games, II, Annals of Mathematics Studies, 28, 1953, 307-317.

[10] L. S. Shapley, Cores of convex games, Int. J. of Game Theory, 1, 1971, 11-26. 